\section{TRACKING OF ADHD-LIKE SYMPTOMS FROM CHILDHOOD TO ADOLESCENCE IN A GREEK BIRTH COHORT AND ASSOCIATED FACTORS}

\author{
A. Palili ${ }^{1}$, A. Veltsista ${ }^{1}$, I. Vassi ${ }^{1}$, A. Gika ${ }^{1}$, \\ G. Kolaitis ${ }^{2}$, C. Bakoula ${ }^{1}$
}

11st Department of Paediatrics, ${ }^{21}$ st Department of Child Psychiatry, Aghia Sophia Children's Hospital, University of Athens, Athens, Greece

Aim: To explore the prevalence of inattention, hyperactivity and impulsivity (ADHD-like symptoms) at the ages of 7 and 18 and associated factors in a representative Greek population.

Methods: Information was derived from a populationbased sample of 11,048 newborn infants, followedup at 7 and 18 years, through questionnaire surveys. After applying a matching procedure, a data set of 2,695 individuals covering all the three time periods was compiled and used in this study. Pearson $X^{2}$ test and multivariate logistic regression analysis were used to assess associations between ADHDlike symptoms and various perinatal and childhood factors.

Results: In childhood, the prevalence of hyperactivity was $7 \%$, inattention $9.5 \%$ and impulsivity $7 \%$, while a substantial decline of these symptoms was observed at 18 years. The male to female ratio was 3 to 1 . Adverse perinatal factors, poor academic performance, comorbidity, physical punishment and frequent report of accidents and conflicts with peers were found to be associated with ADHD-like symptoms at 7 years. The factors found to be associated with ADHD-like symptoms during adolescence were gender, maternal smoking during pregnancy, maternal stress and physical punishment during childhood.

Conclusion: The tracking of ADHD-like symptoms in the Greek population and the associated factors coincided with the international trends of this disorder.

Recommendations to decrease adverse perinatal outcomes, maternal smoking during pregnancy, maternal stress and physical punishment of the child should be evaluated in future longitudinal investigations.

\section{LET'S TALK ABOUT SEX: RESEARCH AND DEVELOPMENT OF THE NEW BOARD GAME FOR ADOLESCENTS WITH CHRONIC CONDITIONS: SECZ TALK}

\author{
A. van Staa ${ }^{1,2}$, H.A. van der Stege ${ }^{1}$, \\ On Your Own Feet
}

${ }^{1}$ Expertise Centre Transitions of Care, Rotterdam University, ${ }^{2}$ Institute Health Policy \& Management, Erasmus University Rotterdam, Rotterdam, The Netherlands

Background: The development of sexuality and intimate relations is often delayed in adolescents with chronic conditions. Although discussing sexuality is part of good transitional care, providers in paediatric health care often feel unprepared for it.

\section{Aims:}

1) To research current practice, attitudes and preferences of adolescents with chronic conditions, their parents \& paediatric care providers toward addressing sexuality during medical / nursing conversations.

2) To develop an attractive intervention to facilitate communication.

\section{Methods:}

1) Observations of 31 consultations \& web-based survey for all chronic patients (12-19 yrs) of a paediatric hospital \& their parents; followed by a national survey for providers of adolescent health care;

2) Participatory approach to develop intervention.

Results: Observations of medical consultations revealed that non-medical issues are rarely addressed during medical consultations. This was confirmed in a survey among 1039 adolescents and 778 parents. Fifty percent find it important to discuss fertility and sexuality in relation to the chronic condition, but this topic is almost never covered. Over $75 \%$ of 159 surveyed healthcare providers in rehabilitation centres and hospitals find this topic important, but need practical tools to facilitate the discussion. With the help of experts, healthcare providers and young people, a new board game -SeCZ Talk- was developed and pilottested in small groups. All users were very positive about the game, that develops knowledge, attitude 